\title{
Controlling the coupling between localized and delocalized surface plasmon modes in a metallic photonic crystal slab
}

\author{
A. Christ ${ }^{1,2}$, T. Zentgraf ${ }^{1}$, J. Kuhl ${ }^{1}$, N. A. Gippius ${ }^{3}$, S. G. Tikhodeev ${ }^{3}$, O. J. F. Martin ${ }^{2}$, and H. Giessen ${ }^{4}$ \\ ${ }^{I}$ Max-Planck-Institut für Festkörperforschung, 70569 Stuttgart, Germany \\ ${ }^{2}$ Nanophotonics and Metrology Laboratory, Swiss Federal Institute of Technology, 1015 Lausanne, Switzerland \\ ${ }^{3}$ A. M. Prokhorov General Physics Institute RAS, Vavilova 38, Moscow 119991, Russia \\ ${ }^{4} 4^{\text {th }}$ Physics Institute, University of Stuttgart, 70550 Stuttgart, Germany \\ Phone: ++41-21-693-3968,email: andre.christ@epfl.ch
}

\begin{abstract}
We study the influence of controlled coupling between localized and delocalized surface plasmon modes on the transmission properties of metallic photonic crystals. The presented interaction phenomena are important for the optimization of future plasmonic nanodevices.

(C)2006 Optical Society of America

OCIS codes: (240.6680) Surface plasmons; (260.3910) Metals, optics of; (050.2770) Gratings
\end{abstract}

Although metallic photonic crystal slab structures have been investigated for quite some time, only little effort has been devoted to the investigation of more complex sample geometries. With regard to the optimization of future plasmonic nanodevices, especially the systematic study of metallic structures which simultaneously support localized and delocalized surface plasmon modes in the same energy range seems very important. In extension to recent work on periodically arranged spherical void structures [1], we therefore present a detailed discussion of coupling between localized and delocalized surface plasmon modes in a novel multilayer metallic photonic crystal design. The analysis is based upon optical transmission measurements as well as numerical simulations using a scattering-matrix formalism [2]. In particular, we show that the surface plasmon dispersion and hence the optical transmission properties can be controlled by varying the geometrical parameters (e.g., metal film or dielectric spacer layer thickness) of the metallic slab structure.
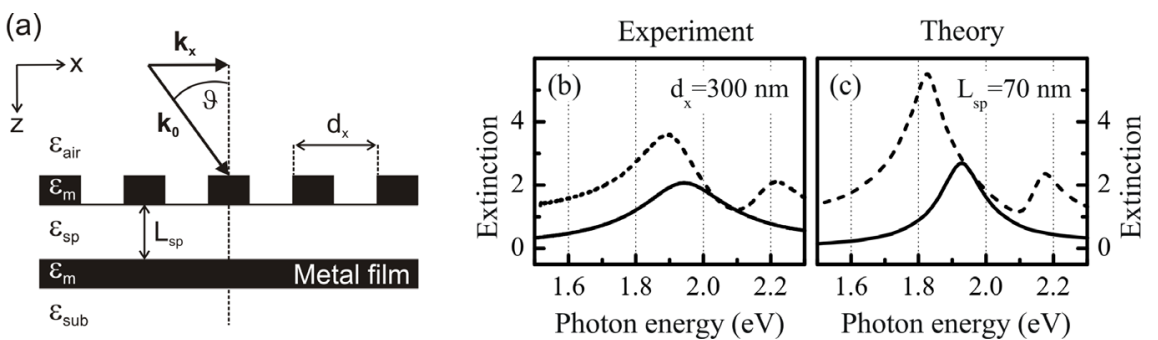

Fig. 1. A schematic view of the investigated sample structure is shown in panel (a). The gold nanowire grating and the nearby silver film are separated by a dielectric spacer layer of thickness $\mathrm{L}_{\mathrm{sp}}$. Experimentally and theoretically obtained extinction spectra are displayed in panels (b) and (c). Exemplary, spectra of a pure gold grating (solid lines) and of the metallic multilayer structure $\left(\mathrm{L}_{\mathrm{sp}}=70 \mathrm{~nm}\right.$, dashed lines) are compared in both panels. The nanowire cross-section $(100 \times 15$ $\left.\mathrm{nm}^{2}\right)$ and the grating period $\left(\mathrm{d}_{\mathrm{x}}=300 \mathrm{~nm}\right)$ have been kept constant. The spectra are shown for TM polarization and normal light incidence.

The schematic view of the metallic photonic crystal slab design is depicted in panel (a) of Fig. 1. The model structure consists of a one-dimensional gold nanowire array and a 20 -nm-thick silver film which are spatially separated by a $\mathrm{SiO}_{2}$ spacer layer. The specific sample geometry therefore allows both the optical excitation of nanowire particle plasmons as well as Bragg-induced surface plasmon modes at the nearby metal film.

Exemplarily, transmission spectra of a bare gold grating without additional silver film (solid lines) are compared with the results of the analyzed multilayer photonic crystal design (dashed lines). Experimental as well as theoretical results are displayed in panels (b) and (c) of Fig. 1, respectively. Instead of a single nanowire-related extinction maximum (i.e., bare grating), the spectra of the multilayer photonic crystal are characterized by two pronounced resonances. The redshifted extinction maximum at $1.85 \mathrm{eV}$ arises due to coupling of the localized wire plasmon and its own mirror-induced image. Additionally, the Bragg- or grating-induced short-range surface plasmon mode of the thin silver film shows up as a second maximum at approximately $2.2 \mathrm{eV}$. We show that especially strong coupling between these resonances becomes the dominat interaction effect. Similar to the recently discussed phenomenon of 


\section{QMF1.pdf}

waveguide-plasmon polariton formation in metallo-dielectric photonic crystal slabs [3], plasmon-plasmon interaction leads to a remarkable splitting when both plasmon modes are tuned into resonance.
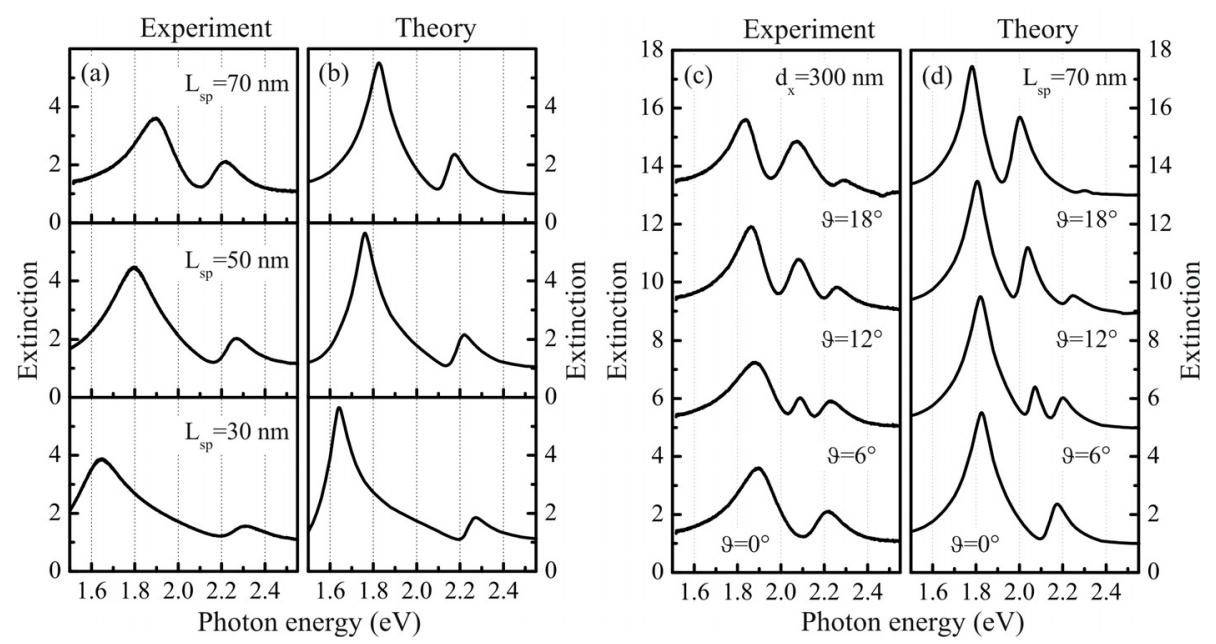

Fig. 2. Measured and calculated extinction spectra of the metallic photonic crystal slab (TM polarization) are shown in dependence on the spacer layer thickness and in dependence on the light incidence angle. Spectra for structures $\left(\mathrm{d}_{\mathrm{x}}=300\right.$ $\mathrm{nm}$ ) with a spacer layer thickness of $30 \mathrm{~nm}, 50 \mathrm{~nm}$, and $70 \mathrm{~nm}$ are compared in panels (a) and (b). Additionally, the angular dependence is analyzed in panels (c) and (d). From bottom to top, the angle $\vartheta$ is increased from $0^{\circ}$ to $18^{\circ}$ in steps of $6^{\circ}$. The spacer layer thickness of $70 \mathrm{~nm}$ and the grating period of $300 \mathrm{~nm}$ both remain unchanged. The individual spectra are shifted upwards for clarity.

The spacer layer dependent transmission spectra of the metallic photonic crystal slab are displayed in panels (a) and (b) of Fig. 2. A reduction of the spacer layer thickness directly influences the spectral position of both resonances. For example, the lower localized mode is strongly redshifted due to the increased image-induced coupling. It is important to remark that the coupling between the nanowire plasmon resonance and its own image results in a socalled magnetic resonance. Such a magnetic resonance might be of importance, especially for a possible realization of negative refractive index metamaterials. Furthermore, a clear angle dependent anticrossing behavior due to polariton formation can be observed in panels (c) and (d) of Fig. 2. While the lower antisymmetric band edge surface plasmon mode is optically inactive for measurements at normal incidence, it shows up as a third resonance at central position under inclined illumination. Note that the excitation efficiency of the upper symmetric short-range mode is reduced when shifting to higher energies for increased incidence angles. Simultaneously, the antisymmetric mode is redshifted and couples to the localized nanowire plasmon resonance. Hence, its excitation efficiency is strongly increased due to mixing of the localized and delocalized plasmon modes.

In conclusion, we have experimentally and theoretically shown that the coupling between localized and delocalized surface plasmon modes will strongly modify the optical transmission properties of metallic photonic crystal slab structures. The observed interaction phenomena result in polariton formation and hence an avoided crossing of the supported plasmon resonances. More generally, the proposed model system introduces a new approach to plasmon engineering and metamaterials with negative refractive index, which is of great importance both from fundamental as well as from applicational point of view.

We acknowledge support from DFG (SPP1113 and FOR557) and BMBF (FKZ 13N8340/1).

[1] T. A. Kelf, Y. Sugawara, J. J. Baumberg, M. Abdelsalam, and P. N. Bartlett "Plasmonic Band Gaps and Trapped Plasmons on nanostructured Metal Surfaces", Phys. Rev. Lett. 95, 116802 (2005).

[2] S. G. Tikhodeev, A. L. Yablonskii, E. A. Muljarov, N. A. Gippius, and T. Ishihara, "Quasiguided modes and optical properties of photonic crystal slabs", Phys. Rev. B 66, 045102 (2002).

[3] A. Christ, S. G. Tikhodeev, N. A. Gippius, J. Kuhl, and H. Giessen, "Waveguide-plasmon polaritons: Strong coupling of photonic and electronic resonances in a metallic photonic crystal slab", Phys. Rev. Lett. 91, 183901 (2003). 